\title{
Propuesta metodológica de un modelo de Benchmarking para centros de investigación y desarrollo tecnológico $(\mathrm{I}+\mathrm{D})$ del sector petrolero $^{* 1}$
}

\section{Methodological Proposal of a Benchmarking Model for Technological Development Research Centers (R \& D) in the Oil Sector}

Nilsson Javier Martine* Pérez ${ }^{* * 2}$

Universidad Santo Tomás e Icontec, Bogotá, Colombia

\section{RESUMEN}

Los modelos de excelencia planteados por algunos países, a través de sus premios nacionales a la gestión de la calidad, son considerados un instrumento impulsor de la competitividad empresarial, que se fundamentan principalmente en la gestión integral de la estrategia, los procesos y la cultura. Este último elemento busca asegurar para las organizaciones un proceso de aprendizaje continuo que es apalancado en una gran proporción por el Benchmarking. De acuerdo con esto, el Benchmarking es considerado hoy día un elemento clave de la gestión integral empresarial, debido a que su aplicación estructurada, sistémica y continua facilita y promueve el aprendizaje organizacional y el mejora-
Recibido: 26 de noviembre de 2009 Revisado: 14 de febrero de 2010 Aceptado: 12 de marzo de 2010

* Artículo de investigación científica resultado del proyecto de investigación.

** Correspondencia: Nilsson Javier Martínez Pérez. Especialista en Administración y Gerencia de Sistemas de Gestión de Calidad y Magíster en Calidad y Gestión Integral. Correo electrónico: nilsson.martinez@ecopetrol.com.co

miento continuo de las organizaciones que se enfrentan a un entorno que cambia rápida y permanentemente.

Con base en lo anterior, las organizaciones del sector petrolero en el ámbito internacional han puesto en evidencia la necesidad de contar con un modelo de Benchmarking para su gestión de $\mathrm{I}+\mathrm{D}$, que les permita determinar con eficacia cuáles son los factores críticos de éxito para asegurar, identificar, adaptar e implementar, así como las mejores prácticas que garanticen los niveles de desempeño deseados para dicha gestión.

El presente artículo propone una metodología para un modelo de Benchmarking para la gestión de I+D, 
el cual surge de la recopilación y análisis de información y experiencias en el tema por parte de algunos centros de I+D del sector petrolero, identificados y seleccionados como de clase mundial; adicionalmente, pretende servir de guía a los centros de I+D de este sector que deseen implementar un proceso lógico y sencillo de Benchmarking para la gestión de I+D de su organización, mediante la aplicación de unas etapas y herramientas básicas que son planteadas para ello.

Palabras clave: benchmarking, centros de I+D, modelos metodológicos, factores críticos de éxito, mejores prácticas, sector petrolero.

\section{ABSTRACT}

Excellence models formulatded by some countries through their national awards for quality management are considered an instrument promoting business competitiveness, and they are primarily based on the comprehensive management strategy, processes and culture. This last element seeks to ensure organizations a continuous learning process which is leveraged in a large proportion by the Benchmarking. Accordingly, Benchmarking is now considered a key element of integrated business management because its structured, systematic and continuous development and promotion of organizational learning and continuous improvement of organizations facing a constantly and fast changing environment.

Based on the foregoing, the international oil sector organizations have highlighted the need for a Benchmarking model for $\mathrm{R} \& \mathrm{D}$ management to enable them to effectively determine what are the critical factors of success to ensure, identify, adapt and implement, and the best practices to ensure desired levels of performance for this management.

This article proposes a methodology Benchmarking model for the R \& D management, which arises from the compilation and analysis of information and experiences on the subject by some of the world class identified and selected R \& D centers in the oil sector; in addition, this article aims to guide the R \& D centers in this sector who wish to implement a simple and logical process of Benchmarking to manage their $\mathrm{R} \& \mathrm{D}$, through the implementation of some basic steps and tools for this.

Key Words: Benchmarking, R\&D centers, methodological models, critical success factors, best practices, oil sector.

\section{INTRODUCCIÓN}

La gestión de la investigación y el desarrollo tecnológico $(\mathrm{I}+\mathrm{D})$ de una organización es considerada en la actualidad (especialmente por las organizaciones de clase mundial del sector petrolero) como la gestión causante de la sostenibilidad del negocio en el largo plazo y del aumento sustancial de su competitividad.

Las organizaciones han venido preocupándose en buena medida por asegurar el éxito de la gestión de la I+D mediante una adecuada articulación con la estrategia del negocio y una efectiva gestión de los proyectos de I+D, entre otros aspectos; no obstante, y para poder lograr esto han tenido que realizar esfuerzos adicionales para configurar sistemas de inteligencia competitiva que les permitan tomar decisiones confiables, mediante la obtención y el análisis de información estratégica que no podría lograrse de manera adecuada sin la integración de herramientas clave como la vigilancia tecnológica, la prospectiva tecnológica y el Benchmarking .

Para realizar la propuesta metodológica del modelo de Benchmarking estructurado, sistémico y continuo para la gestión de I+D se llevó a cabo un estudio de investigación enfocado a conocer y comprender los modelos de Benchmarking que son implementados por algunos de los centros de investigación y desarrollo tecnológico 
(I+D) del sector petrolero; de esta manera se conoció la metodología de Benchmarking implementada, la importancia del Benchmarking, cómo se determinan los factores críticos de éxito que deben ser referenciados y qué tipo de esquemas de cooperación con otros centros de I+D vienen siendo utilizados, entre otros aspectos.

Esta propuesta permitirá a centros de I+D del sector petrolero (como es el caso del Instituto Colombiano de Petróleo de Ecopetrol S.A., para quien se formuló esta investigación) establecer nuevos indicadores que reflejen de manera contundente el componente innovador de su gestión de investigación y desarrollo tecnológico (I+D), así como indagar por mejores prácticas de sus procesos, productos y servicios en otros centros de I+D del sector.

Para dar inicio a la investigación se identificaron y caracterizaron las treinta y nueve (39) primeras empresas del ranking oficial del sector petrolero PIW "Petroleum Intelligence Weekly" (incluyendo sus respectivos cen- tros de I+D). Esta caracterización permitió conocer, entre otros aspectos, sus actividades principales, los avances tecnológicos, la infraestructura y los servicios. Posteriormente, a través de la participación de seis (6) de estos centros de I+D en el diligenciamiento de una encuesta virtual, desarrollada especialmente para este estudio y aplicada por red, fue posible verificar algunas de las características y métricas claves del desempeño en la gestión de I+D.

Luego se hizo un recorrido adecuado por los fundamentos básicos y conceptuales que permiten contextualizar nacional e internacionalmente el "Benchmarking" como una de las competencias prioritarias a ser aseguradas dentro de una organización empresarial. Para tal fin se estableció el siguiente mapa conceptual (Figura 1) que permite visualizar en forma global cada uno de los aspectos clave para el desarrollo y el entendimiento del contexto del presente trabajo de investigación.

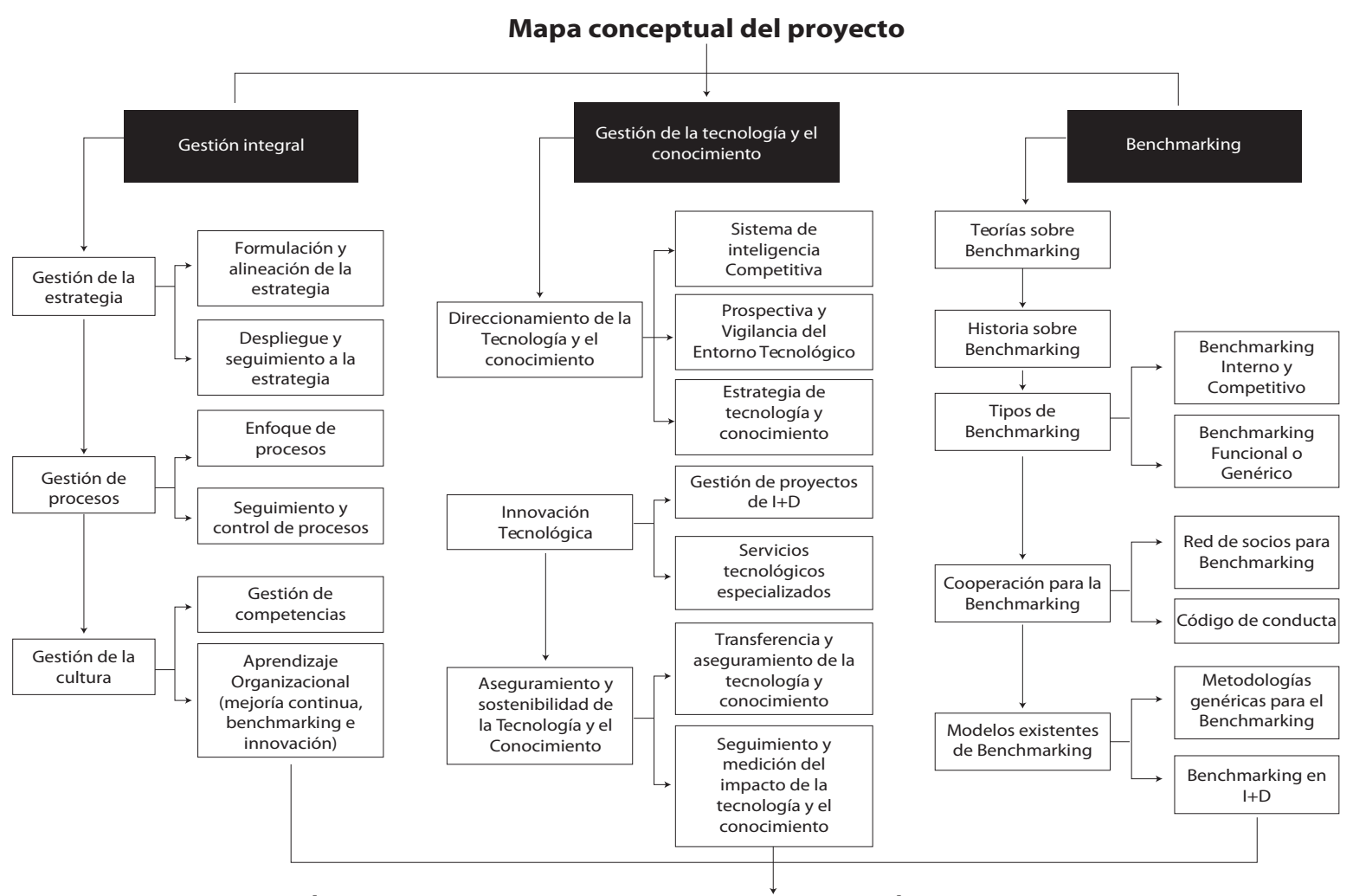

PROPUESTA METODOLÒGICA DE MODELO DE BENCHMARKING PARA LA GESTIÓN DE I+D DE UNA EMPRESA PETROLERA

Figura 1. Mapa conceptual de la investigación.

Fuente: Elaboración propia. 


\section{METODOLOGÍA}

Para el desarrollo de la investigación se estableció seguir la metodología de Benchmarking de Spendolini (1994), por considerarla práctica, sencilla y completa ${ }^{1}$ Basados en el análisis de otros modelos de Benchmarking existentes, tales como los modelos de APQC (American Productivity and Quality Center), John Deere, Robert Camp y el marco teórico y conceptual de este trabajo, se observó que ésta era una metodología que podría aplicarse de manera genérica a cualquier producto, servicio o proceso, como punto de partida para ser aplicada a la gestión de I+D.
Por otra parte, y con miras a la recopilación de información, se aplicó la técnica de la perspectiva distributiva y estructural planteada por Ibáñez en su trabajo de las perspectivas de la investigación social ${ }^{2}$. En consecuencia, se aplicó una encuesta como parte de las técnicas distributivas a través del diseño de un cuestionario y un dispositivo de análisis que permitió conocer de manera ágil y oportuna el contexto actual de los centros de I+D seleccionados respecto de sus modelos de Benchmarking. También se hizo uso de la aplicación de técnicas como las de análisis estructural de textos, entrevistas en profundidad y grupos de discusión, a partir los que se complementa la investigación. En la figura 2 se presenta en forma esquemática la metodología seguida:

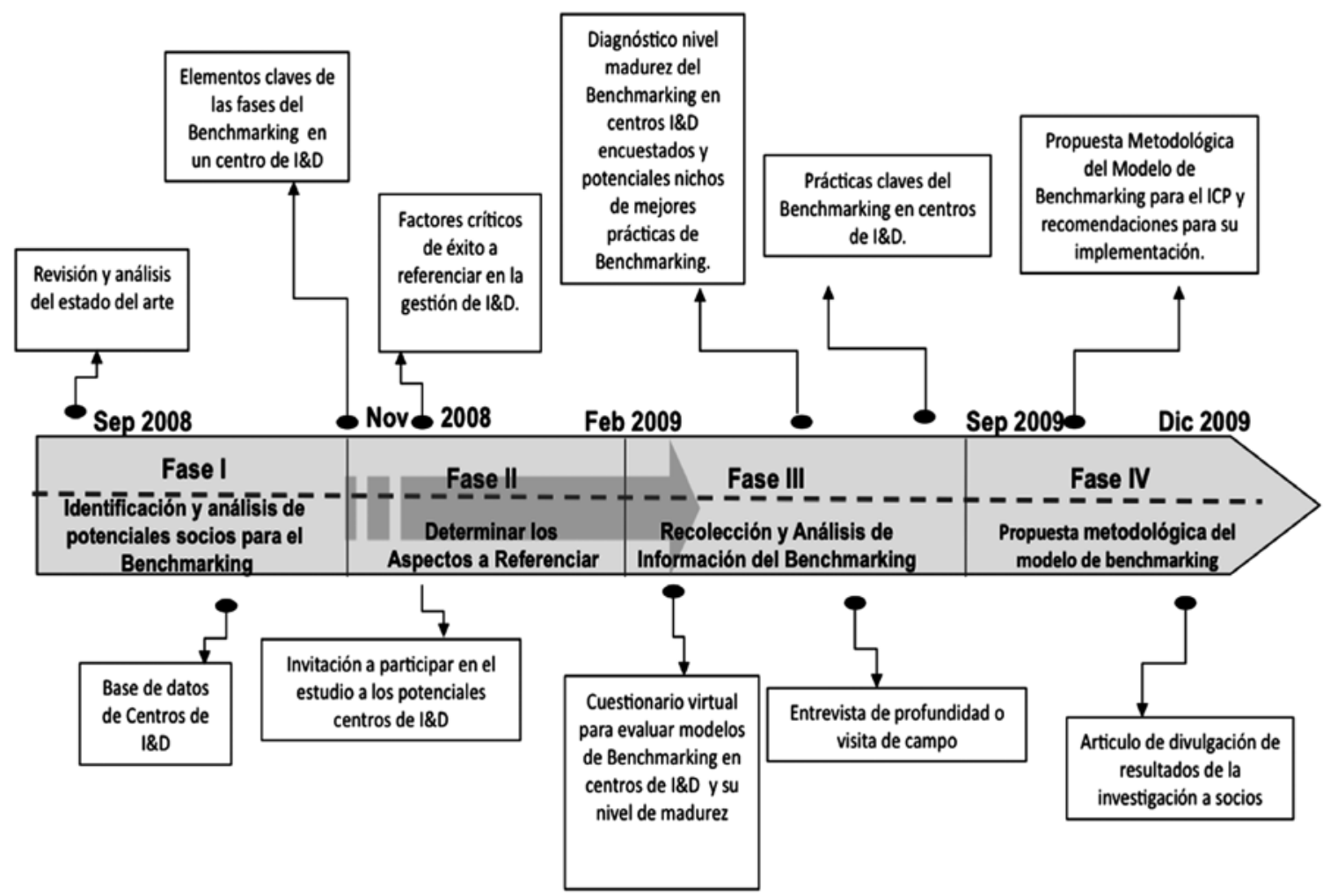

Fiǵura 2. Metodología de investigación

Fuente: Elaboración propia.

1 SPENDOLINI, Michael. Benchmarking. Bogotá, D.C. : Norma, 1994. $248 \mathrm{p}$.
2 IBÁÑEZ, Jesús. Perspectivas de la investigación social: el diseño en las tres perspectivas. En: GARCÍA FERRANDO, Manuel; IBÁNEZ ALONSO, Jesús y ALVIRA MARTÍN, Francisco (Comps.). El análisis de la realidad social. Métodos y técnicas de investigación. Madrid : Alianza, 1990. p. 49 -83. 
Fase I: Identificación y análisis de potenciales socios para el Benchmarking

\section{Revisión y análisis del estado del arte}

Inicialmente se estableció un grupo de discusión en el que se intercambiaron ideas a partir del análisis individual y estructural de artículos e información pertinente al tema de Benchmarking en I+D. Dentro de los temas centrales que fueron abordados pueden mencionarse:

- Las fases del proceso de Benchmarking según Spendolini.

- El Benchmarking en I+D y el desarrollo de herramientas de Benchmarking dentro de los proyectos de I+D.

- Los sistemas de medición en I+D.

- El Benchmarking en institutos de I+D.

- Los cambios en la gestión estratégica de la tecnología.

- Los indicadores de I+D.

- Los planes estratégicos en institutos de investigación y desarrollo tecnológico.

- Las tendencias en I+D.

\section{Bases de datos de los centros de investigación y desarrollo tecnológico}

Se identificaron y caracterizaron las treinta y nueve (39) primeras empresas (incluyendo sus respectivos centros de I+D) del ranking oficial del sector petrolero PIW "Petroleum Intelligence Weekly". Esta caracterización permitió conocer, entre otros aspectos, sus actividades principales, sus avances tecnológicos, su infraestructura y sus servicios. Las empresas con las que Ecopetrol
S.A. se debe comparar son aquellas que según el ranking del PIW se encuentran ubicadas por encima de la posición 27, que pertenecen al sector del petróleo y gas, que han establecido algún tipo de relación con Ecopetrol y que desarrollan proyectos de I+D.

\section{Elementos clave de las fases del Benchmarking}

Después de revisar cada una de las fases planteadas por Spendolini para el Benchmarking, se identificaron los aspectos a considerar en cada una de las fases que se muestran a continuación:

Fase 1: Qué se va a referenciar - selección del proyecto

- Identificar el tipo de Benchmarking requerido.

- Identificar el alcance del estudio del Benchmarking

- Identificar losfactores críticos de éxito a referenciar.

- Identificar el tipo de información a recopilar.

Fase 2: Formación de un equipo de Benchmarking - planeación del proyecto

- Identificar los aspectos a tener en cuenta durante la formación del equipo.

- Identificar y seleccionar los miembros del equipo Benchmarking con base en unos criterios establecidos.

- Capacitar al personal que conforma el equipo de Benchmarking.

- Verificar y medir el cumplimiento de asignaciones. 
Fase3: Identificacióndelos socios del benchmarking

- Identificar lo mejor de su clase para establecer su red de contactos.

- Identificar las mejores prácticas a referenciar.

Fase 4: Recopilación y análisis de información que sirve de referencia

- Establecer una metodología para recopilar información de referencia.

- Identificar sus mejores prácticas para comparar con otros.

Fase 5: Actuar - implementación de mejores prácticas

- Realizar un informe con los resultados del estudio sobre información que sirve de referencia para los socios o para la organización.

- Priorizar la implementación de las mejores prácticas evidenciadas.

- Implementar las mejores prácticas y medir su desempeño.

- Comprobar beneficios.

\section{Fase II: Determinación de los aspectos a referenciar}

\section{Factores críticos de éxito a "referenciar" en la gestión de $I+D$}

Desde este punto de vista, lo primero que se debe hacer para realizar un buen proceso de Benchmarking es identificar los factores críticos de éxito de la gestión a "referenciar", los cuales en nuestro caso en particular se analizaron, a partir de: 1) la lectura del libro de Spendolini, en el que se reconocieron potenciales factores críticos de éxito para la gestión de I+D realizada por centros como los que son objeto de estudio y que se encuentran consolidados en la base de datos desarrollada para este estudio, y 2) los resultados de las reuniones empresariales en las que se identificaron los riesgos a los que está expuesta la empresa.

Inicialmente, se seleccionaron algunos factores críticos de éxito desde la información consolidada en la base de datos, estos fueron:

- Capital intelectual.

- Servicios técnicos especializados.

- Transferencia y aseguramiento del conocimiento y de la tecnología.

- Indicadores y/o métricas de desempeño en la gestión I+D.

- Gestión de proyectos de I+D.

- Habilidades y competencias en I+D.

- Incentivos y reconocimientos en I+D.

- Estrategia tecnológica.

- Alianzas estratégicas.

- Proyectos de investigación y desarrollo tecnológico.

- Participación de potenciales empresas o centros de $\mathrm{I}+\mathrm{D}$.

\section{Participación de las potenciales empresas 0 centros de $I+D$}

De la base de datos desarrollada se seleccionaron aquellas empresas clave para este estudio. Se contac- 
tó formalmente a cada uno de los representantes de dichas empresas o centros I+D con el fin de presentarles el objetivo del proyecto, de conocer la forma como los centros de $\mathrm{I}+\mathrm{D}$ están realizando sus ejercicios de Benchmarking, cuál modelo de Benchmarking aplican, cuáles son las mejores prácticas sobre él y, finalmente, realizar un análisis comparativo de las métricas de desempeño más críticas de la gestión de I+D y darles a conocer el beneficio obtenido al participar en la investigación.

La estrategia utilizada fue inicialmente a través de correos electrónicos, en los que se adjuntaba una carta oficial de invitación a las empresas o centros de I+D y la cual en forma concreta describió el objetivo del proyecto, los requerimientos del estudio, preguntas sobre la capacidad y disposición de la empresa para compartir información del proceso objeto del estudio, requisitos de tiempos para entrega de información, descripción de los beneficios por participar en el proyecto y del nivel de confidencialidad de la información recibida.

En total se contactaron formalmente por correo electrónico las 39 primeras empresas relacionadas en el ranking del PIW y los centros de I+D, de las cuales 6 empresas contestaron positivamente sobre la posibilidad 0 interés en participar.

\section{Fase III: Recolección y análisis de información del Benchmarking}

A partir del mapa conceptual definido en la Figura 1 se determinó la estrategia de desarrollo de la presente investigación, consolidada en una matriz categorial que sirvió como punto de partida para analizar cada uno de los aspectos conceptuales que fundamentan el desarrollo del cuestionario virtual planteado.

\section{Cuestionario virtual para evaluar modelos de Benchmarking en centros de $I+D$ y su nivel de madurez}

Con base en la matriz categorial, en los factores críticos de éxito seleccionados y siguiendo cada una de las fases para el desarrollo de un estudio común de Benchmarking de acuerdo con lo propuesto por Spendolini, se definió una estructura de encuesta en tres partes:

Primera parte: Información general

Segunda parte: Análisis de cada fase del Benchmarking según Spendolini

- Determinar a qué se le va a hacer Benchmarking: "selección del proyecto".

- Formar un equipo de Benchmarking: planeación del proyecto.

- Identificar los socios del Benchmarking.

- Recopilar y analizar información de Benchmarking.

- Actuar: "Implementación de mejores prácticas".

Tercera Parte: Análisis de métricas en la gestión I+D.

La metodología de Spendolini $i^{3}$ sirvió de base para estructurar la encuesta virtual que permitió, como primer paso, sondear la percepción de cada uno de los referentes con respecto al nivel de madurez que tienen en cada una de las fases, pudiendo de esta manera consolidar un diagnóstico del nivel de madurez en la realización del Benchmarking en I+D. 
Como características del cuestionario virtual, se observa que: 1) fue desarrollado en el sistema MySQL por ser fácil, compatible con otros sistemas y libre. 2) el acceso a la herramienta virtual se hizo a través de la Web de Ecopetrol S.A. utilizando el link: http://200.4.21.21/ I+D/ y cuenta con dos plataformas, una de acceso al módulo de participante y otra de acceso como administrador. 3) el cuestionario fue diseñado en español y en inglés considerando que gran parte de los invitados manejan este último idioma.

\section{Entrevista de profundidad o visita de campo}

Con base en la matriz categorial y de acuerdo con las mejores prácticas identificadas, una vez revisadas y analizadas las respuestas al cuestionario virtual, se diseñó y realizó una entrevista en profundidad para algunos de los centros de I+D seleccionados, que permitió indagar más su modelo de Benchmarking. Esta entrevista tuvo por objeto corroborar, identificar y comprender mejores prácticas de los centros de $\mathrm{I}+\mathrm{D}$ encuestados con respecto a temas específicos, como vigilancia y planeación tecnológica, gestión de proyectos $\mathrm{I}+\mathrm{D}$, transferencia de tecnologías, conocimientos y propiedad intelectual.

Inicialmente, se contactó por correo electrónico y a través del canal oficial entre Ecopetrol S.A. y cada una de las empresas mencionadas, a los empleados que participaron en el cuestionario y con quienes ya se tenía establecida comunicación, con miras a obtener la autorización para realizar la visita o entrevista en profundidad.

\section{Fase IV: Propuesta metodológica del modelo de Benchmarking}

Una vez finalizada la tercera fase de la metodología se realizó la propuesta metodológica del modelo de Benchmarking para centros de $\mathrm{I}+\mathrm{D}$ del sector petrolero, propuesta que hace parte de los resultados de esta investigación y que capitaliza las experiencias y mejores prácticas observadas durante la investigación.

\section{RESULTADOS}

A través de la encuesta virtual realizada a cada uno de los centros de I+D objeto del estudio, se logró recopilar información del nivel de madurez que cada uno de éstos posee en las etapas básicas del Benchmarking. Este nivel de madurez fue establecido tomando como criterio base de referencia la metodología de Benchmarking planteada por Spendolinit.

Para lograr lo anterior, y a través de preguntas específicas de la encuesta virtual, cada una de las etapas y aspectos mínimos considerados por la metodología de Spendolini fueron evaluados en los centros de I+D; se les ofreció la oportunidad de que al final de cada etapa ellos mismos definieran en qué nivel de madurez se encontraban. Estos resultados evidencian no sólo la importancia que tiene para los centros de I+D conocer con certeza qué tan adecuadas son las métricas de desempeño que hoy se están aplicando, sino también la necesidad de asegurar mecanismos de comparación permanentes de aquellas métricas de desempeño comunes, determinar la posición competitiva e implementar las mejores prácticas. En la tabla 1 se refleja el resumen de los resultados obtenidos.

4 SPENDOLINI, Op. Cit. 


\section{Métricas de desempeño más importantes a resaltar del grupo de centros de $\mathrm{I}+\mathrm{D}$, objeto del estudio}

- Inversión en proyectos de investigación y desarrollo.

- Patentes solicitadas y aprobadas por año.
- Divulgación técnico-científica a través de publicaciones.

- Alianzas estratégicas.

- Proyectos de investigación y desarrollo tecnológico al año.

Tabla 1. Determinación del nivel de madurez de los centros de I+D del sector petrolero

\begin{tabular}{|c|c|c|}
\hline $\begin{array}{c}\text { Fases de la } \\
\text { metodología de } \\
\text { Spendolini }\end{array}$ & Hallazgos & Diaǵnóstico \\
\hline $\begin{array}{c}\text { Cómo determinar } \\
\text { a qué hacer } \\
\text { Benchmarking }\end{array}$ & $\begin{array}{l}\text {-Todos los participantes identificaron a su or- } \\
\text { ganización como el único cliente para quienes } \\
\text { formalizan los resultados de los estudios de } \\
\text { Benchmarking. } \\
\text {-Cinco de los seis centros de I+D participantes } \\
\text { han identificado el tipo de Benchmarking para } \\
\text { realizarlo como competitivo. } \\
\text {-Los resultados en su mayoría son utilizados para } \\
\text { realizar estudios comparativos, desarrollar planes } \\
\text { estratégicos, establecer metas y oportunidades de } \\
\text { mejora. } \\
\text {-Los factores críticos de éxito más referenciados } \\
\text { por los participantes fueron: las alianzas estraté- } \\
\text { gicas, el capital intelectual, el aseguramiento del } \\
\text { conocimiento, la gestión de los proyectos de I+D } \\
\text { y la estrategia tecnológica. } \\
\text {-Ninguno de ellos se encuentra actualmente refe- } \\
\text { renciando la inteligencia competitiva. }\end{array}$ & $\begin{array}{l}\text {-Según los resultados obtenidos, los } \\
\text { centros de I+D objeto del estudio } \\
\text { se consideran dentro de un nivel de } \\
\text { madurez medio - bajo con respecto } \\
\text { a esta fase. } \\
\text {-Algunas de las recomendaciones } \\
\text { se enfocan para realizar siempre un } \\
\text { Benchmarking interno o funcional } \\
\text { antes de efectuar Benchmarking } \\
\text { competitivo. } \\
\text {-De los seis participantes, según su } \\
\text { propia percepción, uno se consideró } \\
\text { en un nivel alto con respecto a esta } \\
\text { fase, dos se consideran en un nivel } \\
\text { medio, uno en un nivel bajo y dos de } \\
\text { ellos se abstuvieron de dar respuesta. }\end{array}$ \\
\hline
\end{tabular}




\begin{tabular}{|c|c|c|}
\hline $\begin{array}{c}\text { Cómo conforman } \\
\text { los equipos de } \\
\text { Benchmarking }\end{array}$ & $\begin{array}{l}\text {-Algunos hacen partícipes del equipo de } \\
\text { Benchmarking a sus empleados, otros lo confor- } \\
\text { man con expertos internos y otros realizan sus es- } \\
\text { tudios de Benchmarking con el apoyo de expertos } \\
\text { externos; pero ninguno de los participantes del } \\
\text { estudio conforma su equipo considerando repre- } \\
\text { sentatividad de los tres (3) tipos de integrantes. } \\
\text {-Para seleccionar el personal que hace parte del } \\
\text { equipo, seleccionaron los siguientes criterios en } \\
\text { orden de importancia: experiencia, disponibilidad, } \\
\text { habilidad de comunicación y credibilidad. } \\
\text {-Ninguno de ellos consideró el espíritu de equipo } \\
\text { como uno de los criterios de selección importante. } \\
\text {-En relación con la frecuencia de capacitación del } \\
\text { equipo en Benchmarking, dos de ellos no respon- } \\
\text { dieron, dos de ellos realizan capacitaciones anua- } \\
\text { les y los dos restantes cada dos años. } \\
\text {-En términos generales, la necesidad de realizar } \\
\text { estudios de Benchmarking surge como una nece- } \\
\text { sidad puntual dentro de alguna de las áreas opera- } \\
\text { tivas de los negocios, pero ninguno tiene estruc- } \\
\text { turado un cronograma sistémico para realizar este } \\
\text { tipo de estudios. }\end{array}$ & $\begin{array}{l}\text {-Según los resultados obtenidos, los } \\
\text { centros de I+D objeto del estudio } \\
\text { se consideran dentro de un nivel de } \\
\text { madurez medio - bajo con respecto } \\
\text { a esta fase. } \\
\text {-Se evidenció la necesidad de confor- } \\
\text { mar sus equipos de Benchmarking } \\
\text { con la participación de perso- } \\
\text { nal experto técnico en I+D y de } \\
\text { Benchmarking que asegure el co- } \\
\text { rrecto desempeño del estudio, apo- } \\
\text { ye la coordinación, dirija los esfuer- } \\
\text { zos de los integrantes que llevan a } \\
\text { cabo la mayor parte de las tareas de } \\
\text { Benchmarking. } \\
\text { - Se selecciona el equipo sobre la } \\
\text { base de los criterios de habilidad y } \\
\text { motivación, descartando aquellos } \\
\text { como conveniencia, disponibilidad y } \\
\text { personas prescindibles. }\end{array}$ \\
\hline $\begin{array}{l}\text { Identificación } \\
\text { de socios para el } \\
\text { Benchmarking }\end{array}$ & $\begin{array}{l}\text {-En su mayoría, los centros de I+D participantes } \\
\text { del estudio identifican en orden de importancia a } \\
\text { los mejores de su clase a través de: los rankings } \\
\text { oficiales de la industria, reconocimientos, pre- } \\
\text { mios especiales, alianzas estratégicas y asesores } \\
\text { externos. } \\
\text {-Identifican mejores prácticas en otros centros de } \\
\text { I+D principalmente por medio de encuestas, bases } \\
\text { de datos y entrevistas, aunque algunos consideran } \\
\text { también internet y los medios de comunicación. }\end{array}$ & $\begin{array}{l}\text {-Los participantes del estudio, con- } \\
\text { cuerdan en su mayoría en encontrar- } \\
\text { se en un nivel de madurez medio, } \\
\text { se puede establecer que todos los } \\
\text { participantes tienen un enfoque } \\
\text { claro de cómo desarrollar esta etapa } \\
\text { para obtener resultados importantes } \\
\text { y valiosos. } \\
\text {-Lo anterior refleja que ésta es una } \\
\text { de las fases del Benchmarking más } \\
\text { sólida y madura del grupo de centros } \\
\text { de I+D participantes del estudio. }\end{array}$ \\
\hline
\end{tabular}




\begin{tabular}{|c|c|c|}
\hline $\begin{array}{l}\text { Recopilan y analizan } \\
\text { la información del } \\
\text { Benchmarking }\end{array}$ & $\begin{array}{l}\text {-Los métodos utilizados para recopilar y analizar } \\
\text { información fueron en orden de importancia: pu- } \\
\text { blicaciones, medios de comunicación, la web, visi- } \\
\text { tas a otros centros de I+D, entrevistas telefónicas } \\
\text { y finalmente las encuestas. } \\
\text {-En la mayoría de los casos los centros de I+D } \\
\text { objeto del estudio identifican sus mejores prác- } \\
\text { ticas principalmente a partir de los niveles de } \\
\text { desempeño. }\end{array}$ & $\begin{array}{l}\text {-Con respecto a esta fase, la percep- } \\
\text { ción de los participantes fue en tér- } \\
\text { minos generales media. } \\
\text {-Estos resultados permiten estable- } \\
\text { cer que esta es otra de las fases del } \\
\text { Benchmarking sólida y madura en el } \\
\text { grupo de centros de I+D que partici- } \\
\text { pan en el estudio. }\end{array}$ \\
\hline Actuar & $\begin{array}{l}\text {-Todos los participantes del estudio confirmaron } \\
\text { que entregan un informe al corporativo de su } \\
\text { organización. } \\
\text {-En su mayoría, la prioridad para implementar las } \\
\text { mejores prácticas son según su orden de impor- } \\
\text { tancia: el beneficio económico que se obtenga, el } \\
\text { nivel de innovación, las necesidades actuales, los } \\
\text { recursos y capacidades técnicas que se tengan y el } \\
\text { conocimiento técnico en el tema. } \\
\text {-Los indicadores para medir el desempeño y efec- } \\
\text { tividad del proceso de implementar las mejores } \\
\text { prácticas que fueron seleccionadas en orden de } \\
\text { importancia son: calidad, costo, posición frente a } \\
\text { los referentes y beneficio. } \\
\text {-En términos generales, los participantes capitali- } \\
\text { zan el resultado de referenciar las mejores prácti- } \\
\text { cas, principalmente para la planificación estratégi- } \\
\text { ca, comparar productos y procesos y fijar metas de } \\
\text { desempeño. }\end{array}$ & $\begin{array}{l}\text {-En este sentido la percepción de } \\
\text { los participantes con respecto al ni- } \\
\text { vel de madurez en esta fase fue en } \\
\text { términos generales media-baja. Lo } \\
\text { anterior permite establecer que esta } \\
\text { es una de las fases del Benchmarking } \\
\text { que debe afianzarse en los centros de } \\
\text { I+D que participan en el estudio. }\end{array}$ \\
\hline
\end{tabular}

Fuente: Elaboración propia.

Propuesta metodológica del modelo de Benchmarking para centros de $\mathrm{I}+\mathrm{D}$ del sector petrolero

El estado del arte revela que poseer información estratégica del entorno global es de suma importancia a la hora de pretender obtener y mantener ventajas com- petitivas para una organización, en especial, al referirse a un centro de $\mathrm{I}+\mathrm{D}$ del sector petrolero. Para contar con este tipo de información, afortunadamente existen herramientas modernas como la inteligencia tecnológica, concebida como clave del entendimiento del entorno y de la generación de conocimiento para centros 
de $\mathrm{I}+\mathrm{D}$ que deseen fortalecer su toma de decisiones y la formulación de estrategias encaminadas al desarrollo continuo y sostenible de ventajas competitivas.
El modelo de Benchmarking en I+D propuesto plantea cinco etapas, tal como se observa en la (figura 3).

Figura 3. Método propuesto para el modelo de Benchmarking para la gestión de I+D.

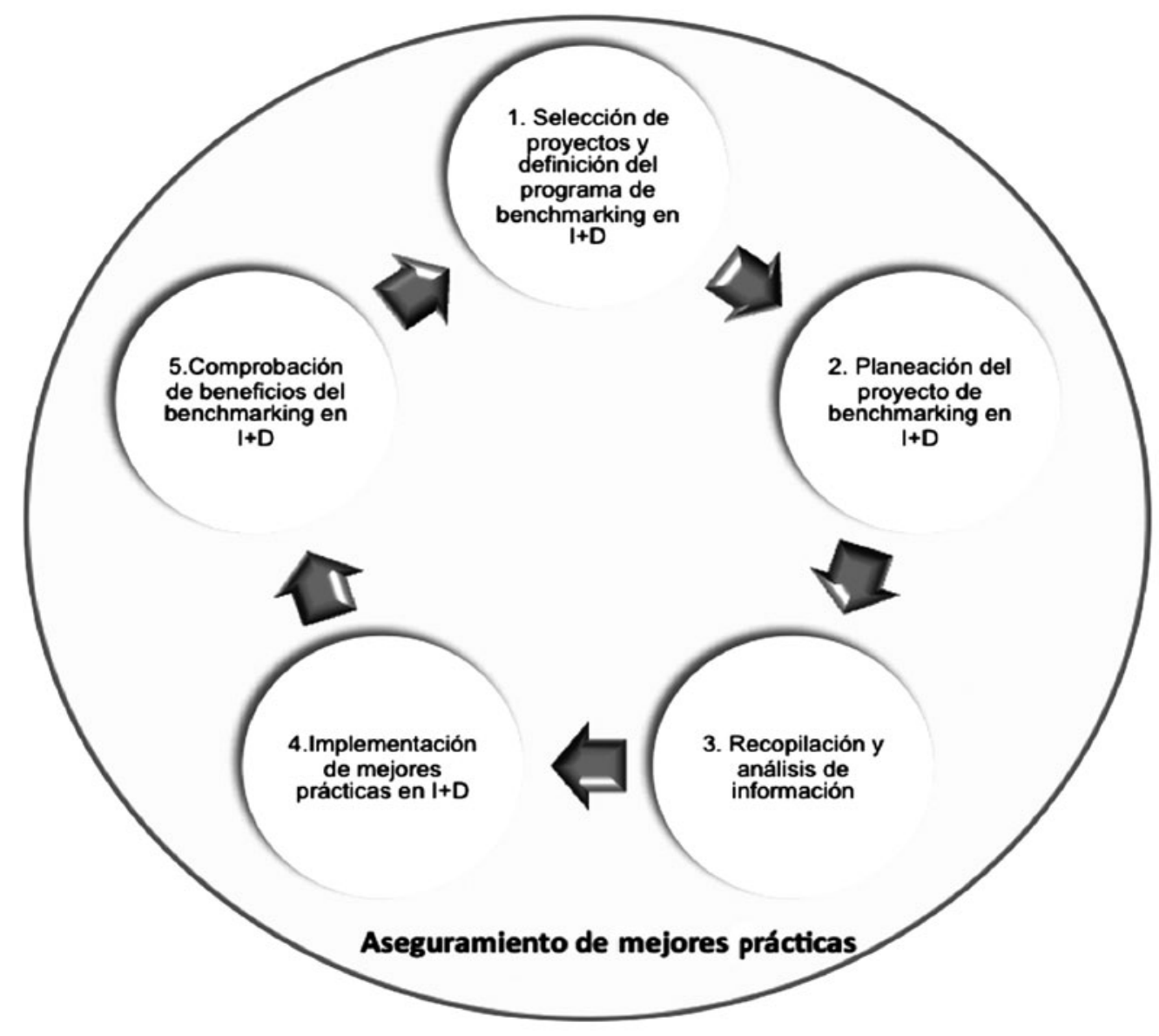

Fuente: Elaboración propia. Etapa 1: Selección de proyectos y definición
del programa de Benchmarking en $I+D$.

Las actividades básicas para realizar durante la selección de proyectos y definición del programa de Benchmarking en I+D son (Figura 4):

- Diagnóstico de necesidades de información de Benchmarking en I+D: para el desarrollo de la eta- pa 1 del modelo metodológico es clave iniciar con la determinación de las necesidades de información de Benchmarking para la gestión de I+D de los usuarios o beneficiarios. Para este modelo de Benchmarking en I+D existen tres tipos de usuarios o beneficiarios clave: 1) El Equipo de Dirección del Centro de I+D; 2) El Equipo de Benchmarking en I+D; 3) Los socios del Benchmarking en I+D. 
De acuerdo con lo anterior, el diagnóstico de necesidades de información del Benchmarking en I+D debe incluir: identificación de los factores críticos de éxito, identificación del tipo de proyecto de Benchmarking a realizar, identificación del tipo de información, uso y cantidad a obtener y definición de la periodicidad del proyecto de Benchmarking en I+D.

- Proyectos de Benchmarking en I+D: con base en el diagnóstico previamente realizado sobre las necesidades de información para el Benchmarking, el equipo de dirección del centro de $\mathrm{I}+\mathrm{D}$ definirá prioridades y determinará el número de proyectos de Benchmarking para la gestión de I+D a realizar. Cada uno de los potenciales factores críticos de éxito evidenciados puede generar un proyecto individual e independiente de Benchmarking que requiere de una adecuada disponibilidad y aseguramiento de recursos humanos, físicos, tecnológicos y financieros. No obstante, debe analizarse la posibilidad de realizar proyectos conjuntos o simultáneos cuando exista un grado de afinidad entre dos o más de los factores críticos de éxito que lo permita, lo cual en algunos casos ayudará a optimizar los recursos. Lo recomendable es no realizar proyectos de Benchmarking en I+D que contemplen más de dos factores críticos de éxito, debido a que el manejo de la información se hace más complejo y se arriesga la eficacia en su recopilación con los socios del Benchmarking.

- Programa de Benchmarking en I+D: el centro de I+D debe establecer, implementar y gestionar un programa efectivo de Benchmarking que, a su vez, contenga todos los proyectos de Benchmarking para la gestión de $\mathrm{I}+\mathrm{D}$ por realizarse durante un periodo de tiempo determinado y dirigidos hacia un propósito específico. El propósito del programa es planear el tipo y número de proyectos de Benchmarking para I+D, e identificar y suministrar los recursos necesarios para realizarlos. El programa de Benchmarking en I+D puede incluir uno o varios proyectos individuales, conjuntos o simultáneos. El equipo de dirección del centro de I+D, debe asignar la responsabilidad y autoridad de dirigir el programa de Benchmarking a su unidad o dependencia más idónea. Las responsabilidades que resultan de esta asignación son: 1) establecer los objetivos y extensión en tiempo del programa de Benchmarking en I+D; 2) establecer las responsabilidades, recursos y procedimientos requeridos para la aplicación del programa de Benchmarking en I+D; 3) asegurar la implementación, seguimiento, medición y mejora del programa de Benchmarking en I+D; 4) llevar los registros apropiados del programa de Benchmarking en I+D.

Fiǵura 4. Primera etapa del método propuesto para el modelo de Benchmarking para la gestión de I+D.

Entradas
- Objetivos estratégicos.
- Métricas de desempeño.
- Información del Entorno
tecnológico.
- Mapa de procesos de la
gestión I+D.

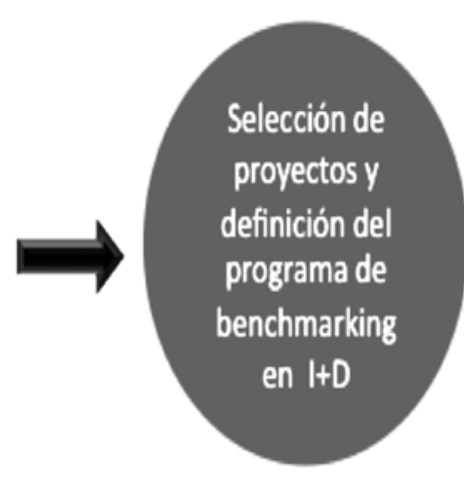

Salidas

- Diagnóstico de necesidades de información de benchmarking en $\mathrm{I}+\mathrm{D}$.

- Proyectos de Benchmarking en $I+D$, con base en factores criticos de éxito. - Programa de benchmarking en $\mathrm{I}+\mathrm{D}$.

Fuente: Elaboración propia. 


\section{Etapa 2: Planeación del proyecto de Benchmarking en $I+D$}

Las actividades básicas por realizar durante la planeación de un proyecto de Benchmarking en I+D son: (Figura 5).

- Definición del equipo de Benchmarking en I+D: por las características del desarrollo de un proyecto de Benchmarking en I+D, es recomendable que el equipo de trabajo sea conformado por representantes de las dependencias o unidades del centro de $\mathrm{I}+\mathrm{D}$ relacionadas con el proyecto de Benchmarking de interés, lo cual contribuye en buena medida a asegurar que las recomendaciones de buenas prácticas resultantes del proyecto sean implementadas por dicha dependencia o unidad. El equipo debe contar con el número suficiente de integrantes que garantice el desarrollo y culminación del proyecto. Este número depende de la complejidad del proceso relacionado con la evaluación y el alcance del proyecto (cuatro a seis personas máximo).

- Análisis del proceso y métricas de desempeño: es de vital importancia que el proceso relacionado con el (los) factor (es) crítico (s) de éxito a evaluar, sea (n) conocido (s) a profundidad, para lo cual se debe tomar su caracterización (definida en el sistema de gestión de calidad del centro de I+D) y revisar dentro del mapa de procesos de la organización a la que pertenece, sus actividades, entradas, salidas, métricas de medio y de resultado, sus metas y recursos. El análisis detallado del proceso le da al equipo de Benchmarking en $\mathrm{I}+\mathrm{D}$ la claridad para definir el propósito y alcance del proyecto. Los aspectos del proceso que se deben tener en cuenta en el análisis son, entre otros, los siguientes: objetivos, proveedores, elementos del proceso (materia prima, equipos, medidas, medio ambiente, métodos y personas), participantes del proceso (quién tiene autoridad sobre el proceso y quién es responsable del mismo), métricas de desempeño del proceso y sus resultados.
- Definición del objetivo y alcance del proyecto de Benchmarking en I+D: el objetivo del proyecto de Benchmarking en I+D debe ser coherente con el diagnóstico de las necesidades de información que fue previamente establecido con los usuarios o beneficiarios de los resultados del Benchmarking en I+D.

Debe establecerse además el alcance del proyecto de Benchmarking en I+D, de forma que se describa su extensión y se confirmen sus límites con base en el diagnóstico de necesidades de información y en términos de los siguientes elementos:

- Etapas de recolección de información requeridas. Definir cuáles de las siguientes etapas son necesarias: investigación secundaria, información cuantitativa, información cualitativa.

- Tipo de Benchmarking a realizar. Definir el tipo de Benchmarking más conveniente de acuerdo con el objetivo definido: Benchmarking interno, competitivo o funcional.

- Ámbito geográfico. Definir si el proyecto tendrá alcance local, nacional, regional (Latinoamérica) 0 mundial.

- Detalle del estudio. Definir el nivel de profundidad en función de la información por recopilar.

- Identificación y selección de los socios para el Benchmarking en I+D: la identificación y selección de los centros de I+D que participan como socios del proyecto de Benchmarking en I+D debe hacerse con base en el análisis detallado de información cuantitativa y cualitativa que permita comparar niveles de desempeño relacionados con el factor crítico de éxito que se desea evaluar, y considerando que los desempeños sean superiores 0 al menos similares a los deseados por el centro de I+D. Para esto es importante utilizar bases de datos de ranking oficiales del sector u otras fuentes clave de información como menciones, premios especiales, medios 
de comunicación, asociaciones profesionales e informes técnicos independientes, que permitan identificar las organizaciones y sus respectivos centros de I+D que se encuentren en las posiciones de desempeño deseadas.

- Elaboración del plan detallado de acción: una vez definido el objetivo y alcance del proyecto de Benchmarking en I+D, y seleccionados los socios que harán parte de él, el equipo asignado debe proceder a diseñar el plan detallado de acción para el proyecto, teniendo en cuenta los siguientes aspectos:

- Objetivo y alcances del proyecto.

- Principales actividades e hitos del proyecto.

- Cronograma.

- Funciones y responsabilidades del equipo de trabajo.

- Presupuesto del proyecto.

- Declaración de asuntos éticos y legales.

Al identificar los recursos necesarios para realizar un proyecto de Benchmarking en $\mathrm{I}+\mathrm{D}$ se deben considerar:

- Recursos financieros necesarios para el desarrollo, implementación, gestión y mejoras a las actividades del proyecto de Benchmarking en I+D.
- El acompañamiento metodológico si se considera necesario.

- Los procesos para lograr y mantener la competencia de los miembros del equipo de trabajo y mejorar constantemente su desempeño.

- La disponibilidad de las personas del equipo y de los expertos que posean la competencia apropiada para el logro de los objetivos propuestos.

- La duración del proyecto de Benchmarking en I+D.

- El tiempo de desplazamiento, el alojamiento y otras necesidades requeridas durante la ejecución del proyecto de Benchmarking en I+D.

Las personas responsables de dirigir el proyecto de Benchmarking en I+D deberán determinar su viabilidad teniendo en cuenta los siguientes factores:

- Información suficiente y apropiada para planificar el proyecto.

- Disponibilidad para la cooperación por parte del socio.

- Disponibilidad de tiempo y de recursos adecuados.

\section{Entradas \\ - Diagnóstico de necesidades de información de Benchmarking en $\mathrm{I}+\mathrm{D}$. \\ - Proyectos de Benchmarking en $I+D$, con base en factores críticos de éxito. \\ - Programa de benchmarking en $1+D$.}

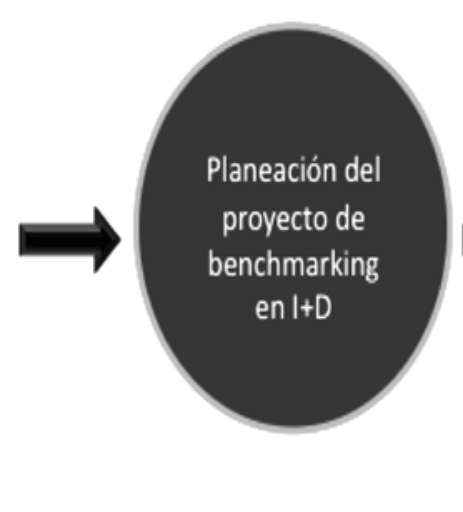

\section{Salidas}

- Equipo de Benchmarking en I+D.

- Proceso y métricas de desempeño analizadas.

- Objetivo y alcance del proyecto de Benchmarking en I+D.

- Socios del Benchmarking en $I+D$, identificados.

- Plan detallado de acción.

Fiǵura 5. Segunda etapa del método propuesto para el modelo de Benchmarking para la gestión de I+D.

Fuente: Elaboración propia. 
Etapa 3: Recopilación y análisis de información del Benchmarking en $I+D$.

Las actividades básicas para realizar durante la recopilación y análisis de información de un proyecto de Benchmarking en I+D son: (Figura 6).

- Determinación de métodos para recopilación de información del proyecto de Benchmarking en I+D: una vez realizada la planeación del proyecto de Benchmarking en I+D y haber clarificado las fuentes de información y los centros de $\mathrm{I}+\mathrm{D}$ a involucrar en el proyecto, se deben analizar y definir los métodos más idóneos de recopilación de información a utilizar.

La tabla 2 muestra la relación existente entre los diferentes métodos de recopilación de información y el medio utilizado para contactar las fuentes o socios del proyecto de Benchmarking en I+D.

Tabla 2. Relación entre métodos de recopilación de la información y medios de contacto.

\begin{tabular}{|l|l|l|l|}
\hline \multicolumn{1}{|c|}{$\begin{array}{c}\text { Métodos de recopilación de la } \\
\text { información }\end{array}$} & \multicolumn{3}{|c|}{ Medio de contacto } \\
\cline { 2 - 4 } & Observación directa & Virtual & Teléfono \\
\hline Encuestas & $\mathrm{X}$ & $\mathrm{X}$ & $\mathrm{X}$ \\
\hline Entrevistas & & $\mathrm{X}$ & $\mathrm{X}$ \\
\hline Visitas o misiones & $\mathrm{X}$ & & \\
\hline Publicaciones & & $\mathrm{X}$ & \\
\hline Revisión de registros archivados & & $\mathrm{X}$ & \\
\hline Participación en eventos & $\mathrm{X}$ & & \\
\hline
\end{tabular}

Fuente: Elaboración propia.

- Definición de la estrategia de recopilación de información del proyecto de Benchmarking en I+D: el propósito de la recopilación de información dentro de un proyecto de Benchmarking en $\mathrm{I}+\mathrm{D}$ es capturar y producir los mejores datos que permitan al equipo de trabajo realizar los análisis de manera eficaz y ser efectivos a la hora de identificar y confirmar las mejores prácticas relacionadas con los factores críticos de éxito que se están evaluando en la gestión de I+D. Independientemente de cuál de los métodos anteriores decida utilizar para la recopilación de la información del proyecto de Benchmarking en $\mathrm{I}+\mathrm{D}$, es recomendable que el equipo de trabajo realice un protocolo de contacto con el socio, asegurando lo siguiente:

- Contar con una lista de los contactos clave (socios del Benchmarking), organizada en orden de prioridad.
- Preparar con antelación el cuestionario de preguntas acordes y organizadas en secuencia lógica para cubrir el tema de interés.

- Coordinar entre el equipo cómo y quiénes contactarán a los socios del Benchmarking. Es preferible que cada socio sea contactado siempre por el mismo miembro del equipo.

- Antes del contacto con un socio, el equipo del proyecto debe informarse y contextualizarse muy bien sobre él.

- Al iniciar el contacto, clarificar quién es y el objeto del contacto que se está realizando. Es importante, además, especificar de manera realista el tiempo requerido y la declaración de confidencialidad que se dará a la información obtenida. 
- Al cerrar el contacto con el socio del proyecto de Benchmarking es importante confirmar datos de contacto de las dos partes (números de teléfono, fax, correo electrónico, etc.) para cualquier necesidad posterior.

- Después de finalizado el contacto, es importante enviar al socio del Benchmarking una nota de agradecimiento por su cooperación con el proyecto y compartirle un resumen de los resultados del contacto realizado.

- Organización y análisis de información recopilada para el proyecto de Benchmarking en I+D: después de haber sido recopilada la información y los datos del proyecto de Benchmarking en I+D, el equipo, a través de los analistas, debe organizar y procesar la información e identificar los potenciales nichos de mejoramiento de los procesos y métricas de desempeño relacionados con el factor crítico de éxito que está siendo evaluado en la gestión de I+D. Para ello es importante realizar como mínimo, los siguientes pasos:

- Consolidar y organizar la información recopilada en una matriz de información normalizada que permita diferenciar el contenido estratégico, operativo, del entorno y económico, entre otros.

- Asegurar el control de calidad de la información consolidada en la matriz.

- Identificar brechas y sus causas, en los diferentes procesos y métricas de desempeño.

Es una buena práctica que en la medida que el equipo del proyecto de Benchmarking en I+D vaya recopilando la información con las fuentes y socios respectivos proceda a organizar dicha información dentro de la matriz de información, clasificándola de la más genérica a la más específica. Asimismo, deben determinarse y verificarse posibles errores de información (transcripción 0 representación equivocada de datos), información que se encuentra desviada del alcance del proyecto de Benchmarking de interés, datos que discrepan entre las diferentes fuentes y socios del proyecto.

El análisis de la información recopilada y organizada en la matriz debe, como mínimo, contemplar los siguientes tres aspectos:

- El análisis de los procesos y métricas de desempeño internas del centro de $\mathrm{I}+\mathrm{D}$ relacionados con el factor crítico de éxito que se está evaluando.

- El análisis de los procesos y métricas de desempeño de la fuente 0 socio del Benchmarking relacionados con el factor crítico de éxito que se está evaluando.

- La comparación de los dos análisis anteriores, para identificar las brechas de desempeño y las mejores prácticas asociadas a los mejores desempeños.

La identificación de las brechas es un aspecto de vital importancia para el proyecto de Benchmarking en I+D ya que su identificación permitirá al equipo de trabajo reconocer los nichos de mejora y como consecuencia profundizar sobre esas prácticas que están permitiendo a los centros de I+D, socios del Benchmarking, alcanzar esos mejores niveles de desempeño.

Para poder identificar las causas de las brechas, el equipo del proyecto de Benchmarking en I+D debe como mínimo comparar los flujos de actividades de los procesos de los socios del Benchmarking para identificar las actividades adicionales que están generando los altos desempeños. Adicionalmente, puede ser de gran apoyo para el análisis que el equipo de trabajo utilice diagramas de causa-efecto, también conocido como diagrama de Ishikawa. Al finalizar esta etapa del proyecto de Benchmarking en I+D se debe documentar la información obtenida y realizar un informe con la siguiente información: centros de I+D participantes, un resumen ejecutivo de las prácticas identificadas, metodología utilizada, hallazgos por tema y perfiles de los centros de I+D. 


Entradas
- Equipo de Benchmarking en
I+D.
- Proceso y métricas de
desempeño analizadas.
- Objetivo y alcance del
proyecto de Benchmarking
en I+D.
- Socios del Benchmarking en
I+D, identificados.
- Plan detallado de acción.

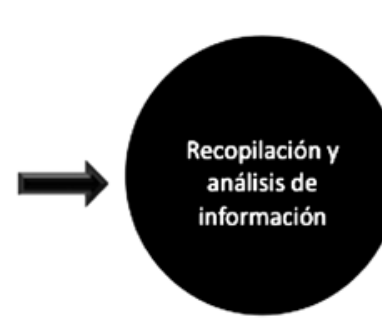

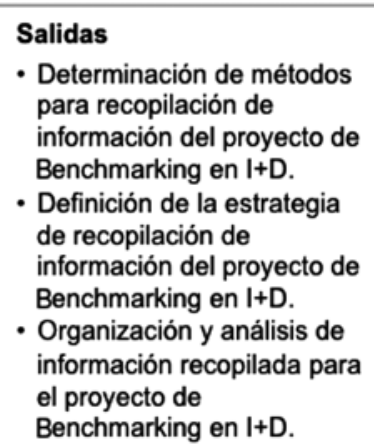

Salidas

- Determinación de métodos Benchmarking en I+D.

Definición de la estrategia el proyecto de Benchmarking en I+D.

Fiǵura 6. Tercera etapa del método propuesto para el modelo de Benchmarking para la gestión de I+D.

Fuente: Elaboración propia.

\section{Etapa 4: Implementación de mejores prácticas en $I+D$}

Las actividades básicas por realizar durante la etapa de implementación de las mejores prácticas de un proyecto de Benchmarking en I+D (figura 7) son:

- Asegurar un plan de implementación y fortalecimiento del conocimiento de la mejor práctica en la gestión de I+D: una vez identificadas las mejores prácticas, sus habilitadores y resultados de desempeño, el equipo del proyecto de Benchmarking en I+D debe realizar análisis adicionales para corroborar si las mejores prácticas pueden ser 0 no adaptadas al centro de I+D.

Para aquellas mejores prácticas que pueden ser adaptadas, se realiza un plan de implementación y de aseguramiento del conocimiento de acuerdo con los lineamientos de la organización a la que pertenece el centro de I+D. Como una buena práctica de esta etapa en el proyecto de Benchmarking en I+D se recomienda que la implementación de la práctica o prácticas seleccionadas consideren en su plan de implementación y aseguramiento seguir las fases de maduración de proyectos o el aseguramiento del conocimiento que haya establecido la organización, el cual debe contemplar como mínimo: objetivos, equipo de trabajo, actividades, procedimientos, cronograma y presupuesto.

- Identificar los indicadores de seguimiento a la implementación y aseguramiento de la mejor práctica en la gestión de I+D: dentro de los indicadores de gestión 0 proceso que más suelen utilizarse para hacer seguimiento a la implementación de una mejor práctica resultante de un proyecto de I+D podemos mencionar el porcentaje de cumplimiento del plan, el índice de ejecución del plan y el nivel de aseguramiento de la práctica, entre otros. Una vez aprobado el plan por parte del equipo de dirección del centro de I+D, debe ejecutarse y hacer seguimiento periódicamente a través de cada uno de los indicadores que se establezcan para ello.

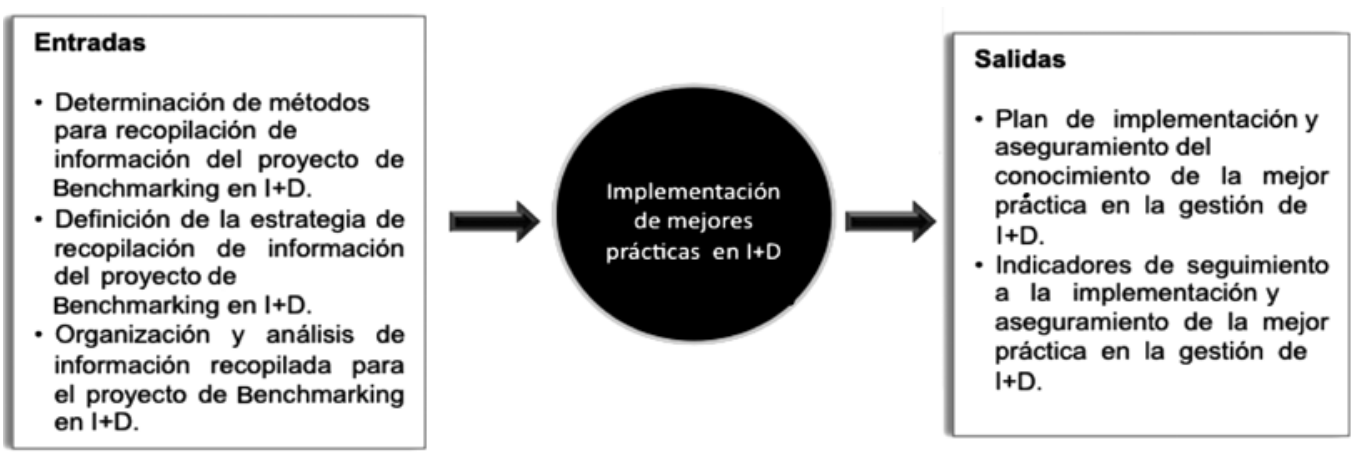

Figura 7. Cuarta etapa del método propuesto para el modelo de Benchmarking para la gestión de I+D.

Fuente: Elaboración propia. 
Etapa 5: Comprobación de beneficios del Benchmarking en $I+D$

El equipo del proyecto de Benchmarking en I+D, a través de su líder de proyecto, debe asegurar que sea evaluado el proceso de la gestión I+D relacionado con la práctica implementada, comparando sus métricas de desempeño antes y después de la implementación de la práctica. Una vez realizada esta comparación, debe retroalimentarse al equipo de dirección del centro de I+D sobre los beneficios generados por la implementación de la mejor práctica resultante del proyecto de Benchmarking en I+D. El equipo del proyecto debe realizar en esta etapa un análisis de lecciones aprendidas que permita mejorar la gestión de los futuros proyectos de este tipo y asegurar, con base en las directrices de retención documental, la memoria técnica y documental generada durante el desarrollo del proyecto de Benchmarking en $\mathrm{I}+\mathrm{D}$.
Es recomendable que el centro de I+D, con base en la periodicidad establecida, realice un nuevo proyecto de Benchmarking que permita identificar la nueva posición de desempeño del centro de I+D en el ranking oficial de su sector industrial. En la medida que el centro de I+D realice Benchmarking periódico sobre el factor crítico de éxito relacionado con la práctica implementada se comprobará la sostenibilidad del conocimiento y los beneficios obtenidos (Figura 8).

Comprobados los beneficios del proyecto de Benchmarking de I+D y la sostenibilidad del conocimiento apropiado a través de la práctica o prácticas implementadas, el centro de I+D por medio del equipo de trabajo del proyecto debe realizar la divulgación de dicho caso de éxito, publicándolo en revistas técnicocientíficas preferiblemente indexadas o mediante ponencias en congresos o eventos nacionales e internacionales de reconocimiento para el sector.
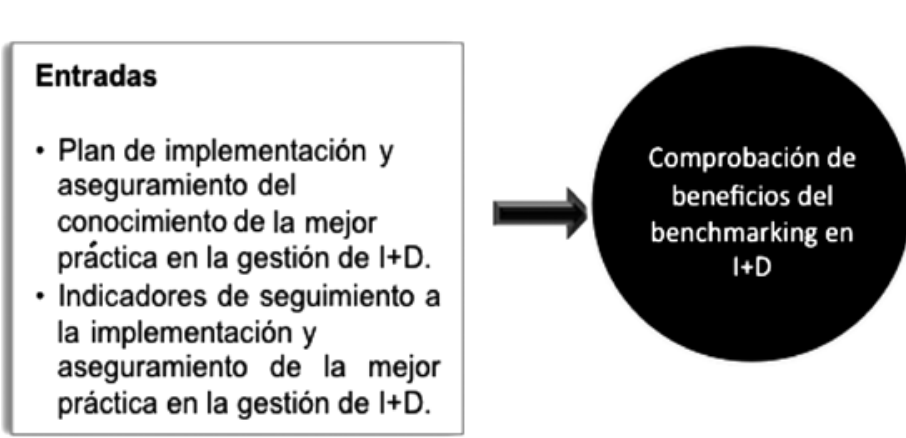

\section{Salidas}

- Comparación del desempeño pre y pos a la implementación de la mejor práctica.

- Presentación de beneficios.

- Lecciones aprendidas del proyecto de benchmarking en I+D.

- Aseguramiento de la memoria técnica y documental.

- Divulgación de caso de éxito.

Fiǵura 8. Quinta etapa del método propuesto para el modelo de Benchmarking para la gestión de I+D.

Fuente: Elaboración propia.

\section{Mejores prácticas identificadas para algunos factores críticos de la gestión de $\mathrm{I}+\mathrm{D}$}

Se deben identificar aquellos factores críticos de éxito que permitan establecer el nivel de contribución que la gestión de $\mathrm{I}+\mathrm{D}$ brinda al negocio de la organización y que permitan establecer parámetros de comparabilidad con otros centros I+D pares. En esta investigación los centros de I+D evaluados seleccionaron con prioridad los siguientes factores críticos de éxito:
- Gestión de proyectos de I+D.

- Gestión del capital humano.

- Gestión de la propiedad intelectual.

- Establecimiento de alianzas estratégicas o convenios de cooperación en I+D. 


\section{DISCUSIÓN}

Analizados los resultados que se obtuvieron a través de la encuesta virtual y la entrevista en profundidad se diagnosticó que todos los centros de I+D objeto del estudio realizan Benchmarking permanentemente, pero no lo hacen de una manera estructurada y sistémica. La realización de los estudios de Benchmarking por parte de los centros de I+D surge, en el mejor de los casos, como respuesta a necesidades puntuales de subsanar el bajo desempeño en alguno de los procesos de la gestión de I+D, y en el resto de los casos simplemente como respuesta a la necesidad de comparar en qué posición de competitividad se encuentran algunas de las métricas de desempeño de la gestión I+D. Tal diagnóstico justifica plenamente realizar esfuerzos ingentes para clarificar y configurar un modelo de Benchmarking en I+D que sea estructurado, sistémico y continuo, que se caracterice por su lógica y por proporcionar herramientas sencillas para planear, ejecutar, verificar y asegurar los diferentes proyectos de Benchmarking que un centro de $\mathrm{I}+\mathrm{D}$ requiera adelantar.

La propuesta metodológica del modelo de Benchmarking en I+D planteada en este trabajo de investigación hace parte integral de un sistema de inteligencia competitiva de la organización que, a su vez, es integrado por tres herramientas de apoyo: el monitoreo y vigilancia tecnológica, el Benchmarking tecnológico y la prospectiva tecnológica.

De igual forma, la propuesta surge de la comprensión de los métodos que los centros de I+D aplican durante la realización de ejercicios de Benchmarking, así como de la necesidad de confirmar cuáles elementos de la gestión de I+D son considerados por esos centros como factores críticos de éxito y de identificar qué prácticas pueden ser consideradas actualmente como mejores de su clase. El método propuesto para el modelo de Benchmarking en $\mathrm{I}+\mathrm{D}$ plantea cinco etapas que son desarrolladas partiendo de insumos clave del centro de I+D, como son: objetivos estratégicos y métricas del desempeño, información del entorno tecnológico y mapa de procesos de la gestión I+D.

Con toda seguridad, la implementación de la propuesta metodológica del modelo de Benchmarking propuesto en este artículo, hará que los centros de I+D del sector fortalezcan su evolución hacia un aprendizaje organizacional continuo. De igual forma, cada vez que se realice la retroalimentación que permita comprobar los beneficios del proyecto de Benchmarking de I+D, se deberá evaluar también la capacidad del equipo de Benchmarking para desarrollar los proyectos, la conformidad con los programas y los cronogramas, la retroalimentación recibida por parte de las áreas del negocio, el tiempo empleado para implementar las prácticas, todo ello con base en la coherencia de los resultados, las tendencias, la conformidad con los planes establecidos, la evolución de las necesidades y las expectativas de las partes interesadas y los registros consolidados.

\section{CONCLUSIONES}

De acuerdo con la información obtenida de los centros de $\mathrm{I}+\mathrm{D}$, objeto de la investigación a través de la encuesta virtual y la entrevista de profundidad se puede concluir que:

a. Las actividades de Benchmarking en I+D surgen en el mejor de los casos como respuesta a necesidades puntuales de subsanar el bajo desempeño en alguno de los procesos de la gestión de I+D, y en el resto de los casos surge simplemente como respuesta a la necesidad de comparar en qué posición de competitividad se encuentran algunas de las métricas de desempeño de la gestión I+D.

b. Todos los centros de I+D consideran las actividades de Benchmarking en $\mathrm{I}+\mathrm{D}$ un elemento clave dentro 
de la gestión integral de su organización y por esta razón, consideran fundamental contar con un modelo estructurado, sistemático y continuo como el que se plantea a partir de este trabajo de investigación, ya que éste va a facilitar y promover el aprendizaje organizacional y, a su vez, el mejoramiento continuo, características que cumple la propuesta metodológica del modelo de Benchmarking para el sector petrolero, resultado de esta investigación.

c. Las actividades de Benchmarking en los centros de $\mathrm{I}+\mathrm{D}$ deben realizarse como un trabajo conjunto con las áreas operativas del negocio en su nivel más estratégico, para que se definan cuáles son las tecnologías prioritarias a incorporar en el corto, mediano y largo plazo, y poder así apalancar el logro de los resultados de la estrategia corporativa.

d. Los centros de I+D reconocen que los beneficios de realizar estudios de Benchmarking se notarán en la medida en que las mejores prácticas identificadas sean implementadas y se mantenga una realimentación sistemática y continua del proceso de Benchmarking en I+D a través del desarrollo de nuevos proyectos que les permitan permanentemente medir su nueva posición de desempeño.

e. Cuatro factores críticos de éxito identificados en esta investigación son los más importantes para los centros de I+D objeto del estudio: gestión de proyectos de I+D, gestión del capital humano, gestión de la propiedad intelectual y establecimiento de alianzas estratégicas o convenios de cooperación en I+D.

Sin embargo, existen otros factores críticos de éxito sobre los cuales se podrían establecer proyectos de Benchmarking con el fin de encontrar otras mejores prácticas.

f. A partir del análisis de las métricas de la gestión de I+D evaluadas en la encuesta virtual se evidenció no sólo la importancia que tiene para los centros de $\mathrm{I}+\mathrm{D}$ conocer con las métricas de desempeño, sino también la necesidad de asegurar mecanismos de comparación permanentes de aquellas métricas de desempeño comunes, determinar la posición competitiva e implementar las mejores prácticas.

g. Pretender igualar la gestión de un centro del sector petrolero con respecto a otro es complicado, ya que cada centro de $\mathrm{I}+\mathrm{D}$ es diferente por naturaleza, la razón de ser de cada uno depende de la estrategia del negocio de la organización a la que pertenece, haciendo que su gestión de I+D sea muy propia. Lo anterior hace que el Benchmarking en I+D tome un grado de complejidad mayor para identificar qué es lo que realmente debe evaluarse 0 referenciarse.

h. La clave para desarrollar un Benchmarking efectivo es comenzar eligiendo áreas o procesos que se encuentren alineados con los objetivos estratégicos de la organización, especialmente aquellos que agregan valor en la toma de decisiones, que impactan directamente el cumplimiento de la estrategia tecnológica y que permiten fijar metas y producir el mayor impacto en la mejora del desempeño del centro de I+D.

La propuesta metodológica del modelo de Benchmarking en I+D obtenida a partir de los resultados de este trabajo de investigación posee las siguientes características:

a. Preserva las etapas básicas de un proceso genérico de Benchmarking, aplicadas de manera específica a la gestión de I+D de una organización del sector petrolero.

b. Se basa en recursos de captación y análisis de información del entorno tecnológico y competitivo, lo que lo hace compatible y sinérgico con el sistema de inteligencia competitiva de un centro de I+D. 
c. Maximiza el aprovechamiento de actividades prácticas, cotidianas y complementarias al Benchmarking dentro de un centro de I+D del sector, como son la participación en congresos, ferias tecnológicas, misiones tecnológicas, prácticas industriales y, en general, eventos empresariales.

e. Viabiliza el desarrollo de cualquier tipo de Benchmarking en $\mathrm{I}+\mathrm{D}$, independientemente de si es liderado por un único centro de $\mathrm{I}+\mathrm{D}$ (individual) 0 si resulta del liderazgo de varios centros de $\mathrm{I}+\mathrm{D}$ que identifican una necesidad y un beneficio común (colaborativo); e independientemente de cuáles centros de I+D harán parte del ejercicio de Benchmarking (interno, competitivo y funcional).

Asegura la sinergia y compatibilidad con el proceso corporativo de Benchmarking de la organización del sector, a la que el centro de $\mathrm{I}+\mathrm{D}$ pertenece.

Asegura la implementación de un proceso continuo y sistemático, mutuamente beneficioso, de análisis y comparación de información clave del desempeño en $\mathrm{I}+\mathrm{D}$, que permite identificar y aplicar permanentemente las mejores prácticas que pueden ayudar a lograr un mejor desempeño competitivo.

\section{RECOMENDACIONES}

- Para la recopilación de información en estudios de Benchmarking en I+D se debe contar con acuerdos de cooperación y criterios de confidencialidad previamente establecidos entre las partes que aseguren la confianza y veracidad de la misma. Dichos acuerdos de cooperación deben estar basados en el principio del mutuo beneficio entre los participantes del proyecto, estableciéndose una guía o código de ética tal que oriente en detalle el comportamiento que el centro de $\mathrm{I}+\mathrm{D}$ y su equi- po de Benchmarking va a tener en las diferentes situaciones del proyecto y que, además, permita definir claramente el manejo de la información, su confidencialidad y el correcto uso de la misma.

- La planeación y destinación de los recursos para ejecutar estos estudios de Benchmarking en I+D debe contar con la participación de todos los niveles jerárquicos dentro del centro de $\mathrm{I}+\mathrm{D}$, desde la dirección, los expertos técnicos y los expertos en Benchmarking, con el fin de establecer objetivos que estén de acuerdo con la estrategia de cada negocio. Es importante tener en cuenta que la influencia positiva del Benchmarking en I+D se extiende más allá del mejoramiento de un proceso en particular, puesto que se promueve también el surgimiento y evolución de una "cultura de aprendizaje" dentro de la organización en todos sus niveles y es un punto clave para el mejoramiento continuo, la calidad total y la competitividad a largo plazo. Dentro de esta planeación se deben considerar, entre otras cosas, el tiempo destinado para el estudio, los recursos disponibles, el personal participante dentro del equipo de trabajo y la información que se requiere.

- Las mejores prácticas que surjan de los estudios de Benchmarking en la gestión de I+D que se realicen deberán ser revisadas con el fin de corroborar su adaptabilidad al centro de I+D, y de esta forma poder realizar un plan de implementación y de aseguramiento del conocimiento, de acuerdo con los lineamientos de la organización a la que pertenece el centro de I+D. Como una buena práctica se recomienda que su implementación cumpla las fases de maduración de un proyecto o el procedimiento que haya establecido la organización para asegurar el conocimiento, y que dicha implementación sea incluida dentro de los planes tácticos y de gestión anuales del centro de I+D, con el fin de facilitar su 
seguimiento periódicamente a través de cada uno de los indicadores que se establezcan para ello.

Finalmente, las mejores prácticas recomiendan realizar estudios de Benchmarking interno dentro de la organización antes de realizar este tipo de estudios con otras entidades, ya que esto facilita determinar falencias, oportunidades de mejora y riesgos, así como focalizar los estudios. Esto permite establecer unos objetivos claros para realizar proyectos de Benchmarking competitivo o funcional a partir de los cuales se puedan encontrar las mejores prácticas que permitan superar dichas brechas. Es mejor conocerse bien a uno mismo antes de intentar conocer a los demás.

\section{BIBLIOGRAFÍA}

ASOCIACIÓNESPAÑOLA DENORMALIZACIÓN Y CERTIFICACIÓN. UNE 166000:2006. Gestión de la $\mathrm{I}+\mathrm{D}+\mathrm{i}$. Terminología y definiciones de las actividades de I+D+i. Madrid : Autor, 2006 p. 12.

ASOCIACIÓN ESPAÑOLA DE NORMALIZACIÓN Y CERTIFICACIÓN. UNE 166001:2006. Gestión de la $\mathrm{I}+\mathrm{D}+\mathrm{i}$. Requisitos de un proyecto de I+D+i. Madrid : Autor, 2006.

ASOCIACIÓN ESPAÑOLA DE NORMALIZACIÓN Y CERTIFICACIÓN. UNE 166002:2006. Gestión de la I+D+i. Requisitos de un sistema de gestión de I+D+i. Madrid : Autor, 2006.

ASOCIACIÓN ESPAÑOLA DE NORMALIZACIÓN Y CERTIFICACIÓN. UNE 166006:2006. Gestión de la I+D+i. Requisitos de un sistema de vigilancia tecnológica. Madrid : Autor, 2006.

ANDERSEN, Bjorn. Industrial Benchmarking for competitive advantage. En: Human Systems Management. Trondheim, Norway. 1999, p. 287-296.
ANTHONY, Robert y GOVINDARAJAN, Vijay. Sistemas de control de gestión. $10^{a}$ ed. Madrid : McGraw-Hill, 2003, p. 768.

ASPRILLA, Diana. Un camino a la competitividad: investigación + desarrollo + innovación. En: Normas \& Calidad. Octubre 8-14, 2007, vol. 22, Fasc. 74. Edición Especial.

CAMP, Robert. Benchmarking. La búsqueda de las mejores prácticas de la industria que conducen a un desempeño excelente. México : Panorama, 1993, p. 336.

CASTELLANOS, Óscar Fernando. Retos y nuevos enfoques en la gestión de la tecnología y del conocimiento. Bogotá D.C. Bio Gestión Programa Interdisciplinario. Universidad Nacional de Colombia. 2008.

CASTELLANOS, Óscar Fernando. Gestión Tecnológica: De un enfoque tradicional a la inteligencia. Bogotá D.C. Bio Gestión Programa Interdisciplinario. Universidad Nacional de Colombia. 2008.

COLOMBIA, MINISTERIO DE COMERCIO, INDUSTRIA Y TURISMO. Guía para las Organizaciones: Premio Colombiano a la Calidad de la Gestión. Bogotá D.C. : Corporación Calidad. 2007.

COLOMBIA, MINISTERIO DE SALUD. Evaluación y ajuste de los procesos, estrategias y organismos encargados de la operación del Sistema de Garantía de Calidad para las Instituciones de Prestación de Servicios. Informe Final Piloto Benchmarking Competitiva. Bogotá D.C. : Consorcio Asociación Centro de Gestión Hospitalaria - Canadian Council on Health Services Accreditation - Qualimed. 2007.

COOMBS, Rod; MCMEEKIN, Andrew y PYBUS, Roger. Toward the development of Benchmark- 
ing tools for R\&D project management. En: R\&D Management, 1998, vol. 28, no 3, p. 175-186.

ECOPETROL S.A. Informe Sistema Integral de Gestión. Bogotá D.C. : Dirección General de Planeación. 2007.

ECOPETROL S.A. Manual de Gestión de Calidad. Bucaramanga: Instituto Colombiano del Petróleo. 2007.

ECOPETROL S.A. Formación de Facilitadores SIGO-PCCG. [diapositivas]. Bogotá D.C. : Corporación Calidad. 2007. 228 diapositivas.

ECOPETROL S.A. Modelo operativo de Ecopetrol. 2007. [diapositivas]. Bogotá D.C.: Unidad de Optimización UOP. 2007. 10 diapositivas.

ECOPETROL S.A. Informe ejercicio interno de evaluación con miras al Premio Interno a la Gestión de la Calidad. [diapositivas]. Bogotá D.C. : Dirección General de Planeación DGP. 2007. 91 diapositivas.

ECOPETROL S.A. Taller de validación Modelo Operativo ICP. [diapositivas]. Bogotá D.C.: Unidad de Optimización UOP. 2007. 5 diapositivas.

ECOPETROL S.A. Diez Principales Riesgos de Ecopetrol. [diapositivas]. Bogotá D.C.: Unidad de Gestión de Riesgos. 2007. 15 diapositivas.

EDLER, Jakob; MEYER, Krahmer y REGER, Guido. Changes in the strategic management of technology: results of a global Benchmarking study. En: R\&D Management. 2002, vol. 32, no 2, p.149-163.

ESCORSA, Pere y VALLS, Jaume. Tecnología e Innovación en la empresa. Dirección y Gestión. Bogotá D.C. : Ediciones UPC, Alfaomega, 2003. 342 p.
ESCORSA, Pere y MASPONS, Ramón. De la vigilancia tecnológica a la inteligencia competitiva. Madrid: Pearson Education, 2001, p. 212. ISBN: 84-205-3057-3.

EUROPEAN INDUSTRIAL RESEARCH MANAGEMENT ASSOCIATION. Benchmarking in R\&D (Working Group 51). París : Autor. 1998.

IBÁÑEZ, Jesús. Perspectivas de la investigación social: el diseño en las tres perspectivas. En: GARCÍA FERRANDO, Manuel; IBÁNEZ ALONSO, Jesús y ALVIRA MARTÍN, Francisco (Comps.). El análisis de la realidad social. Métodos y técnicas de investigación. Madrid : Alianza, 1990, p. 49 - 83.

INSTITUTO COLOMBIANO DE NORMAS TÉCNICAS. Sistemas de gestión de la calidad. Fundamentos y vocabulario. NTC ISO 9000:2000. Bogotá, D.C.: Autor, 2000.

JAKOBIAK, François. Práctica de la vigilia tecnológica. Caracas : Fondo Editorial Fintec. Traducción Francesca Lo Truglio.1995, p. 219.

JEAN-MARIE, Henri. Benchmarking R\&D and companies through patent analysis using free databases and special software: a tool to improve innovative thinking. En: World Patent Information. 2004, no 26, p. 297 - 309.

KAPLAN, Robert. De la estrategia a la creación del valor. Ponencia presentada en Seminario de la Universidad Externado de Colombia, Bogotá D.C. : 2006.

KARLÖF, Bengt y ÖSTBLOM, Svante. Benchmarking: A Signpost to Excellence in Quality and Productivity. [s.l.]: John Wiley \& Sons.1993, p. 208. 
LÓPEZ, Antonio José. Globalización y desarrollo sostenible: efectos ambientales. Coyuntura y Debate. En: Revista Cife. 2003, v. 6. p. 44-57.

MÁRQUEZ, Johanna. Propuesta de un sistema de vigilancia tecnológica para el Instituto Colombiano del Petróleo - ICP. Universidad Industrial de Santander. Bucaramanga. 2008.

NORTON, David y KAPLAN, Robert. Cómo utilizar el cuadro de mando integral para implementar y gestionar su estrategia. Barcelona: Ediciones Gestión, 2000, p. 412.

OCDE/ European Communities. Manual de Oslo. Guía para la recogida e interpretación de datos de innovación. $3^{\text {a }}$ ed. 2005, p. 194.

RAO, Jay. Innovación y tecnología: Clave del crecimiento empresarial. Ponencia presentada en Forum: Formación Ejecutiva en la Universidad de la Sabana, Bogotá D.C., Colombia. 2007.

ROUSSEL, Philip; ERICKSON, Tamara y SAAD Kamal. Tercera Generación de I+D: su integración en la estrategia de negocio. Madrid : McGrawHill, 1991, p. 188.

RUSH, Howard; HOBDAY, Mike; BESSANT, John y ARNOLD, Erik. Strategies for best practice in research and technology institutes: an overview of a Benchmarking exercise. En: R\&D Management.1995, vol. 25, no 1, p. 17-31.

SCHEEL-MAYENBERGER, Carlos. Estrategia de competitividad de la innovación tecnológica. Ponencia en Seminario-Taller Internacional de Marketing, Comercialización y Financiación de Innovación y Tecnologías Estratégicas de las Universidades EAFIT, Nacional de Colombia, Colciencias y la Organización de Estados Americanos OEA, Medellín, Colombia. 2003.

SPEKLE, Roland. Explaining management control structure variety: A transaction cost economics perspective. En: Accounting, organizations and society. 2001, vol. 26, no 4-5, p. 419-441.

SPENDOLINI, Michael. Benchmarking. Bogotá, D.C. : Norma, p. 1994. 248.

VILLAMAYOR, Esteban. Gestión por competencias: una aproximación a su necesidad. VI Reunión sobre Administración de Recursos Humanos de Banca Central. Buenos Aires : mayo 13-14, 2004.

WALKER, Melissa. Cómo escribir trabajos de investigación. Barcelona : Gedisa, 2000, p. 480. 